OPEN ACCESS

Edited by:

Anna Maria Giudetti,

University of Salento, Italy

Reviewed by:

Amilcare Barca,

University of Salento, Italy

Gregory C. Henderson,

Purdue University, United States

*Correspondence:

Tomoyuki Nakano

t-nakano@med.id.yamagata-u.ac.jp

Specialty section:

This article was submitted to Lipid and Fatty Acid Research,

a section of the journal

Frontiers in Physiology

Received: 15 November 2021 Accepted: 07 January 2022

Published: 27 January 2022

Citation:

Nakano T and Goto K (2022) Diacylglycerol Kinase $\varepsilon$ in Adipose Tissues: A Crosstalk Between Signal Transduction and Energy Metabolism.

Front. Physiol. 13:815085.

doi: 10.3389/fphys.2022.815085

\section{Diacylglycerol Kinase $\varepsilon$ in Adipose Tissues: A Crosstalk Between Signal Transduction and Energy Metabolism}

\author{
Tomoyuki Nakano* and Kaoru Goto \\ Department of Anatomy and Cell Biology, School of Medicine, Yamagata University, Yamagata, Japan
}

Diacylglycerol (DG) is unique in lipid metabolism because it serves not only as an intermediate product for triglyceride synthesis, but also as a signaling molecule that activates proteins containing DG-responsive elements, such as protein kinase C. Consequently, DG acts as a hub between energy metabolism and intracellular signaling. Of DG metabolizing pathways, DG kinase (DGK) phosphorylates DG to produce phosphatidic acid, which also serves as a second messenger. Several lines of evidence suggest that DGK is deeply involved in metabolic diseases such as obesity and insulin resistance. Of DGK isozymes, DGKE is simplest in terms of structure, but it is characterized by substrate specificity toward arachidonoyl-DG. Recently, we have reported that $D G K \varepsilon$ deficiency promotes adipose tissue remodeling in mice during the course of high fat diet (HFD) feeding regimen including obesity, insulin resistance, and beige adipogenesis. DGKE ablation engenders altered expression of other lipid metabolizing enzymes, including adipose triglyceride lipase (ATGL), hormonesensitive lipase (HSL), and diacylglycerol acyltransferase (DGAT). Subcellular localization of DGKE in the endoplasmic reticulum suggests involvement of this isozyme in lipid energy homeostasis. This review presents current findings of DGK $\varepsilon$ in lipid-orchestrated pathophysiology, especially unique phenotypes of DGK $\varepsilon$-knockout mice in the early and late stages of obesogenic conditions.

\footnotetext{
Keywords: adipose tissue, adipose triglyceride lipase, beige adipogenesis, diacylglycerol kinase, glucose tolerance, obesity, uncoupling protein 1
}

\section{INTRODUCTION}

Lipid is an indispensable constituent of cells. It composes biological membranes surrounding the cell itself, the nucleus, and subcellular organelles. In addition, lipid is stored as an energy source in a specialized organelle called lipid droplet. Of lipids, diacylglycerol (DG), a basic structure of phospholipids, comprises at least 50 molecular species (Sakane et al., 2018) containing two acyl chains of various combinations at sn-1,2, sn-1,3, or sn-2,3 positions (Zechner et al., 2012). In terms of energy metabolism, DG-containing acyl chains at sn-1 and sn-2 positions (1,2-DG) serves as an intermediate product for triglyceride (TG) synthesis. In terms of signal transduction, 1,2-DG is known to serve as an intracellular signaling molecule that activates several proteins including conventional and novel types of protein kinase C (PKC), Unc-13, RasGRP, and transient receptor potential channels (Kanoh et al., 1990; Nishizuka, 1992; Sakane et al., 2007; Goto et al., 2014). Consequently, DG acts as a hub between lipid metabolism and intracellular signaling. 
As depicted in Figure 1, DG derives from various sources. It is metabolized via several enzymatic pathways. In signal transduction cascade, 1,2-DG derives from phosphatidylinositol 4,5-bisphosphate $\left(\mathrm{PIP}_{2}\right)$, a minor component of biological membrane, via phospholipase C (PLC) upon receptor stimulation (Goto et al., 2007; Blunsom and Cockcroft, 2020). In the course of energy metabolism, DG represents both a precursor of TG synthesis by DG acyltransferase (DGAT) and a product of TG hydrolysis by adipose triglyceride lipase (ATGL) (Zechner et al., 2012). In this regard, the former is 1,2-DG, whereas the latter is 1,3-DG or 2,3-DG. Therefore, these DGs are not intermingled and are separately metabolized. Other DG metabolizing enzymes include the following: (1) Hormone-sensitive lipase (HSL) hydrolyzes 1,3-/2,3-DG to monoacylglycerol (MG); (2) DG lipase $\alpha$ acts on 1,2-DG to produce 2-monoacylglycerol (2-MG); and (3) DG kinase (DGK) phosphorylates 1,2-DG to produce phosphatidic acid (PA). This catalysis is reversed by PA phosphatase, which dephosphorylates PA to generate DG (Goto et al., 2014; Sakane et al., 2018).

Of DG metabolizing enzymes, the significance of DGK has been reviewed comprehensively elsewhere (Sakane et al., 2007; Goto et al., 2014; Mérida et al., 2019). Briefly, DGK comprises an enzyme family composed of 10 isozymes in mammalian species. The DGK isozymes are classified into types $\mathrm{I}-\mathrm{V}$ based on molecular structure. Each DGK isozyme shows a distinct enzymatic property, tissue distribution, and subcellular localization (Goto et al., 2007, 2014; Sakane et al., 2007; Topham and Epand, 2009). Considering these biochemical routes of DG metabolism described above, it is noteworthy that 1,2-DG, a substrate of DGK, lies at the crossroads of both TG precursor and second messenger. This arrangement implicates DGK in the regulation of both energy homeostasis and signal transduction.

Of the DGK family, DGKe is simplest in structure $(64 \mathrm{kDa})$ and belongs to type III DGK (Tang et al., 1996). The most prominent feature of $\mathrm{DGK} \varepsilon$ is its substrate specificity toward sn-2-arachidonoyl (20:4) DG species (Glomset, 1996; Tang et al., 1996; Nakano et al., 2016). Since polyphosphoinositides (PPIns) are composed mostly of sn-1-stearoyl-2-arachidonoyl acyl chains, arachidonoyl-DG is incorporated efficiently into PPIns (Glomset, 1996). However, catalysis of arachidonoylDG by DG lipase $\alpha$ generates 2-arachidonoyl glycerol (2-AG), an endocannabinoid that serves as endogenous "marijuana" in the brain (Bisogno et al., 2003; Tanimura et al., 2010). Therefore DGKE can be reasonably expected to participate in various pathophysiological events. This expectation is supported by several reports describing that $\mathrm{DGK} \varepsilon$ is implicated in kidney diseases (Lemaire et al., 2013; Zhu et al., 2016), seizure (Rodriguez de Turco et al., 2001), inflammatory reaction (Yamamoto et al., 2014), and endoplasmic reticulum (ER) stress (Matsui et al., 2014).

From the perspective of subcellular localization, DGK $\varepsilon$ localizes to the ER (Kobayashi et al., 2007; Matsui et al., 2014; Nakano et al., 2016; Hozumi et al., 2017). Actually, ER is a central site for lipogenesis, from which lipid droplets protrude from the ER membrane stuffed with TG for storage. Therefore, ER-resident DGKe is thought to regulate 1,2-DG for signaling and energy metabolism. Recently, dynamic alterations of adipose tissue physiology under DGKE-deficient conditions have been reported (Nakano et al., 2018, 2020). This review specifically assesses the functional implications of DGK $\varepsilon$ in lipid orchestrated pathophysiology of adipose tissues under short-term and longterm high fat diet (HFD) feeding conditions.

\section{DIACYLGLYCEROL KINASE $\varepsilon$-KNOCKOUT MICE SHOW OBESITY AND GLUCOSE INTOLERANCE UNDER SHORT-TERM HIGH FAT DIET FEEDING CONDITIONS}

To investigate metabolic syndrome, an abnormal lipid metabolism characterized by obesity and insulin resistance, HFD feeding is a useful model (Li et al., 2005; Lee et al., 2011; Benoit et al., 2013; Cantley et al., 2013). This model induces energy stress by overloading a fat-enriched diet, causing excessive TG accumulation in adipose tissues. In normal or healthy individuals, however, these obese conditions can be managed by homeostatic mechanism to keep the organism within a physiological range or at least to avoid immediate deterioration. In this regard, investigating how the phenotype is developed during the course of the model and identifying a primary event of a defect or mutation of a given gene is important because the final stage of metabolic syndrome shows a quite similar phenotype despite the distinct causes.

Nakano et al. (2018) have examined how lipid metabolism is altered in DGKe-knockout (KO) mice under HFD feeding conditions. At 21 days of HFD feeding, a very early phase of obesogenic conditions, DGK $\varepsilon$-KO mice tend to increase more body weight compared with wild-type (WT) mice. At 40 days of HFD feeding, DGK $\mathrm{E}-\mathrm{KO}$ mice show considerable body weight gain and expanded mass of epididymal (i.e., visceral) white adipose tissue (WAT). At cellular level, the adipocyte cell size in DGKE-KO mice increases compared with that of WT controls. Consequently, WAT expansion is ascribed to accelerated lipid overloading in adipocytes of DGKE-KO mice. These results demonstrate that $\mathrm{DGK} \varepsilon-\mathrm{KO}$ mice are prone to obesity during early HFD feeding (Figure 2).

A clue to understanding this phenotype in $\mathrm{DGK} \varepsilon-\mathrm{KO}$ mice might be obtained based on results of glucose tolerance test (GTT) in Figure 2 (Nakano et al., 2018). Regular chow feeding leads to no difference in body weight gain between WT and DGK $\varepsilon-\mathrm{KO}$ mice. It is particularly interesting that in GTT under regular chow feeding, plasma glucose kinetics reveals that glucose tolerance is enhanced in DGKE-KO mice compared with WT controls, although plasma insulin level tends to be lower in DGK $\varepsilon-\mathrm{KO}$ mice. However, results in GTT after 40 days of HFD feeding show the inverse pattern: Glucose excursion is blunted in DGKe-KO mice compared with WT controls. The opposite patterns of glucose clearance in WT and DGKE-KO mice between regular chow and HFD feedings might be explained as follows: glucose uptake is intrinsically facilitated in DGKe-KO mice than WT controls, thereby leading to enhanced TG 


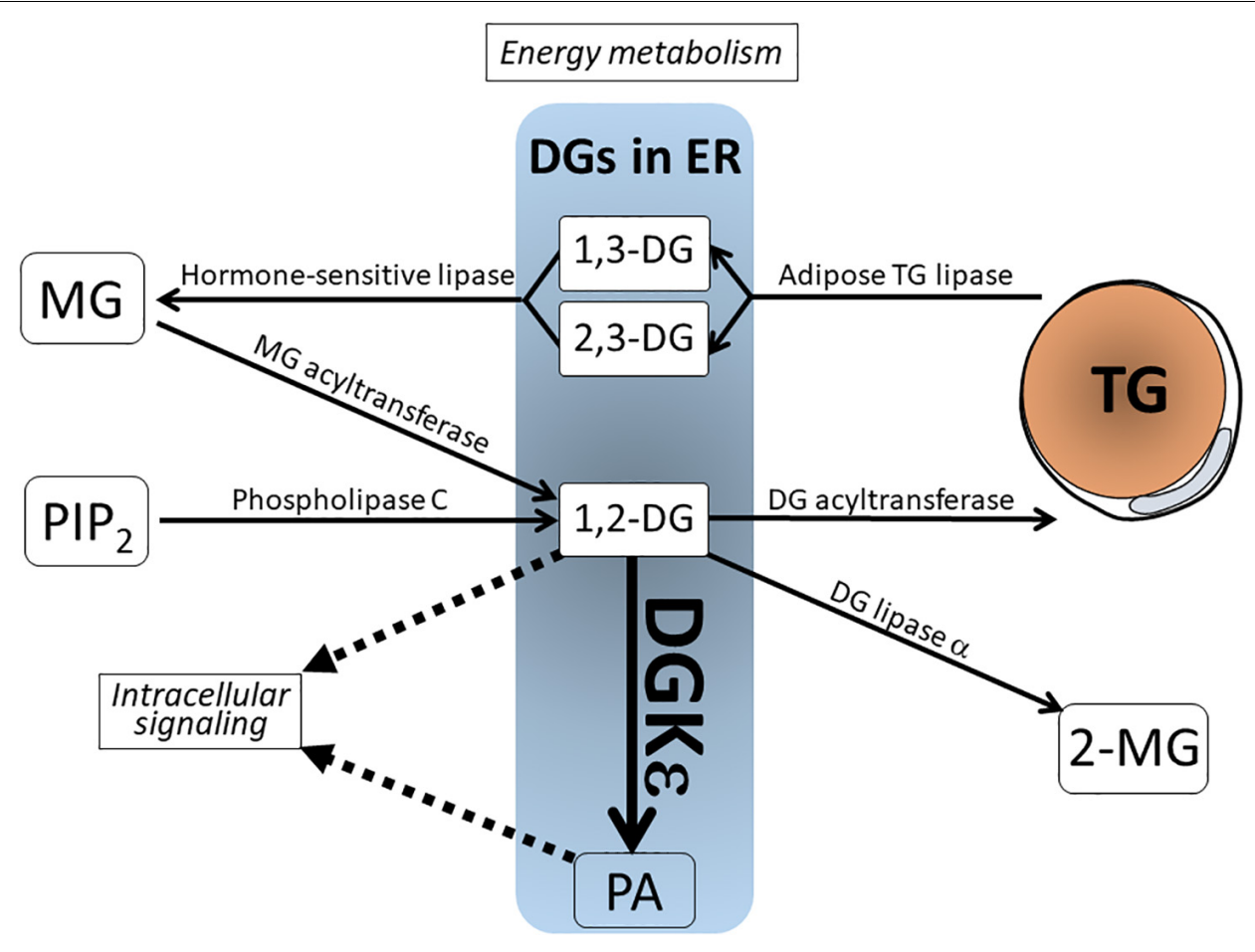

FIGURE 1 | Schematic view of the DG in lipid metabolism and signal transduction. DGs in the endoplasmic reticulum (ER) include various species from distinct sources. 1,2-DG is derived from MG by MG acyltransferase and from phospholipase C-mediated PIP 2 breakdown. 1,3-DG and 2,3-DG are lipolytic products of TG by adipose TG lipase. These DGs are not intermingled and separately metabolized. Note that 1,2-DG serves not only as an intermediate product for TG synthesis, but also as a lipidic second messenger. It is phosphorylated by the action of DGK to produce phosphatidic acid (PA). DG, diacylglycerol; DGK $\varepsilon$, DG kinase $\varepsilon$; MG, monoacylglycerol; PA, phosphatidic acid; PIP 2 , phosphatidylinositol 4,5-bisphosphate; TG, triglyceride; 2-MG, 2-monoacylglycerol.

accumulation under energy excess conditions. This inference is supported by results demonstrating that oleic acid uptake is also facilitated in DGKE-deficient fat tissues (Nakano et al., 2018). Once TG storage exceeds the limit after 40 days of HFD feeding, obesity ensues, thereby causing insulin resistance, as described later.

Recent reports have described that TG overloading in adipocytes coincides with higher rates of basal lipolysis to dissipate extra energy (Wang et al., 2008; Kolditz and Langin, 2010). This machinery is crucially important for the maintenance of whole body energy homeostasis. The TG lipolysis in adipocytes is initiated by adipose TG lipase (ATGL), followed by hormonesensitive lipase (HSL). ATGL catalyzes TG to generate DG, which is cleaved by HSL to release fatty acids. Intermediate product DG in TG lipolytic pathway represents $1,3 / 2,3-\mathrm{DG}$, which is distinct from and which is not intermingled with second messenger 1,2DG (Zechner et al., 2012). However, in TG synthetic pathway, fatty acids are incorporated into MG to generate 1,2-DG, which is acylated immediately into TG by DGAT. This immediate conversion apparently attenuates 1,2-DG because excessive 1,2DG might serve as a second messenger to induce aberrant activation of DG signaling pathway such as PKC. Actually, DGAT expression level is upregulated considerably in WT adipose tissue (Nakano et al., 2018). It is noteworthy that DGAT expression level is induced only slightly in DGKE-deficient adipocytes, which contrasts sharply to WT controls. Taken together, alterations of TG metabolizing enzymes in DGKE-KO mice under shortterm HFD conditions can be summarized as follows: ATGL and HSL expression levels in TG lipolytic pathway are downregulated significantly, whereas the DGAT expression level in TG synthetic pathway is not induced. These alterations engender accumulation of TG and 1,2-DG.

How does TG and 1,2-DG accumulation exert effects on adipocytes? First, overaccumulation of TG in lipid droplets induces an inflammatory reaction in adipose tissues via TNF- $\alpha$ produced by infiltrated macrophages (Cinti et al., 2005; Lumeng et al., 2007). TNF- $\alpha$ facilitates Akt/PKB breakdown, thereby impairing insulin-dependent Akt/PKB signaling (Medina et al., 2005). Second, excessive accumulation of 1,2-DG triggers hyperactivation of DG-sensitive $\mathrm{PKC} \theta$ in adipocytes, thereby increasing serine phosphorylation of insulin receptor substrate-1 (IRS-1). This increase exerts a negative effect on insulin signaling (Kim et al., 2004; Samuel and Shulman, 2012). Collectively, TG and 1,2-DG accumulation exerts "double negative effects" on insulin signaling, thereby inducing insulin resistance in DGKE-deficient adipose tissues under short-term HFD feeding. It must be described that other insulin-reactive organs such as liver and skeletal muscle of DGK $\varepsilon-\mathrm{KO}$ mice exhibit no changes in $\mathrm{PKC}$ and $\mathrm{Akt} / \mathrm{PKB}$ expression and activation status, suggesting that insulin signaling is specifically disturbed in adipose tissues of DGK $\varepsilon-\mathrm{KO}$ mice under short-term HFD feeding conditions. This inference is consistent with results of an earlier study showing 


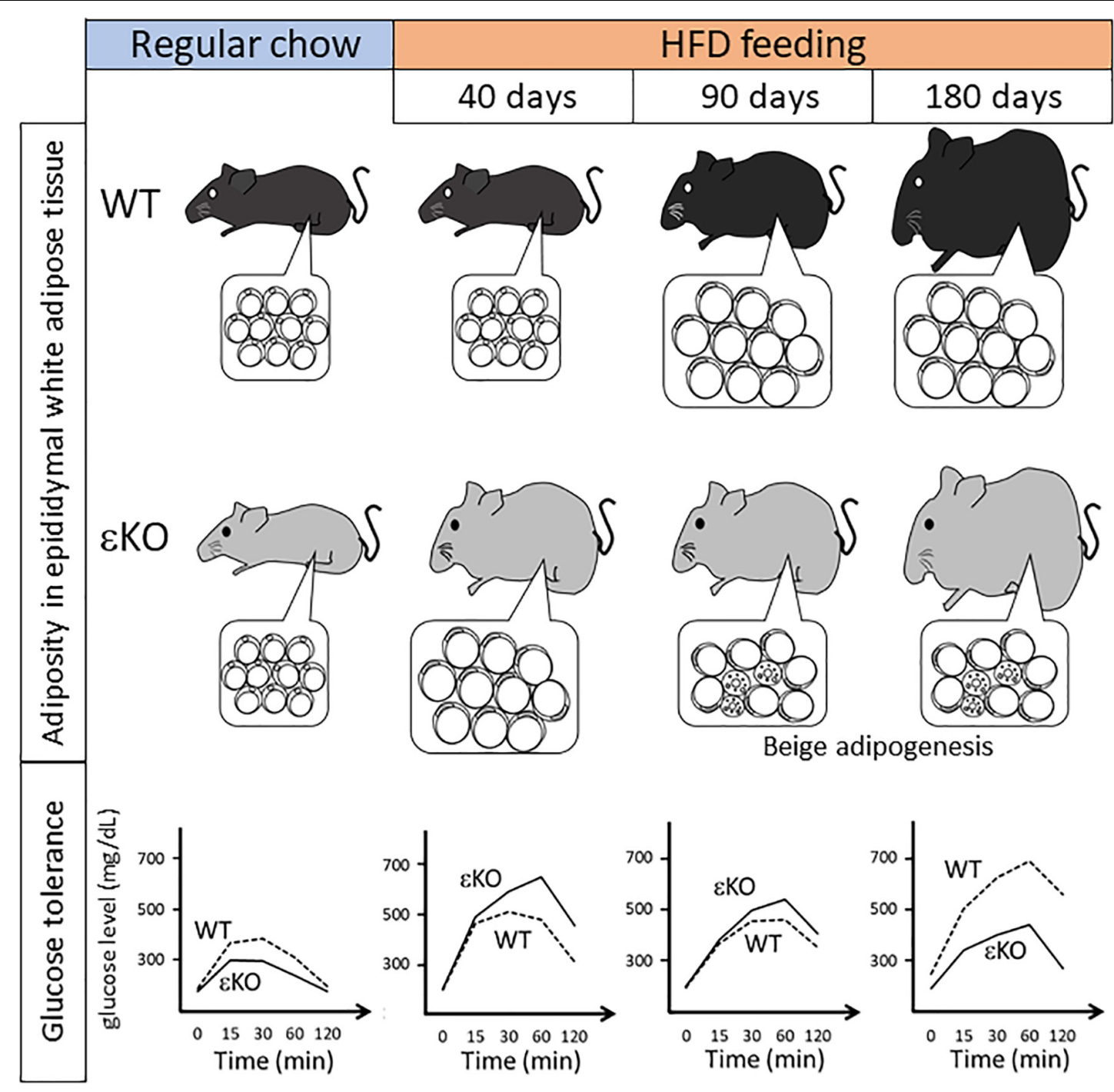

FIGURE 2 | Summary of phenotypical alteration of DGKE-KO mice during the course of HFD feeding regimen. Pathophysiological alterations in body weight, adiposity in epididymal white adipose tissue (insets), and glucose tolerance are shown during the course of HFD feeding (Nakano et al., 2018, 2020). Note that in DGKE-KO mice under long-term HFD feeding beige adipogenesis as shown by multilocular cells is induced, which coincides with improved glucose tolerance. HFD, high fat diet; $\varepsilon \mathrm{KO}, \mathrm{DGK} \varepsilon-\mathrm{KO}$ mice; WT, wild-type mice.

that skeletal muscle insulin sensitivity is unchanged in DGKe-KO mice (Mannerås-Holm et al., 2017).

\section{BEIGE ADIPOGENESIS IS INDUCED IN WHITE ADIPOSE TISSUE OF DIACYLGLYCEROL KINASE $\varepsilon$-KNOCKOUT MICE UNDER LONG-TERM HIGH FAT DIET FEEDING CONDITIONS}

Under short-term (40 days) HFD feeding, a presymptomatic phase of obesity in WT mice, DGKE-KO mice show severe obesity and insulin resistant phenotype, whereas WT mice remain normal. Earlier reports of some studies have described that obese phenotype occurs after 14 weeks of HFD feeding in WT animals (Benoit et al., 2013; Cantley et al., 2013). Next, Nakano et al. (2020) investigated the manner in which this phenotype is changed in DGKE-KO mice under long-term HFD feeding conditions (Figure 2). In WT mice, the obese phenotype becomes evident after 90 days of HFD feeding. The obesity worsens at 180 days. It is particularly interesting that the situation reverses in DGKe-KO mice: During the course of HFD feeding, plasma glucose kinetics of DGKe-KO mice in GTT exhibits the worst pattern at 40 days. At 90 days, however, it improves better and seems similar to that of WT controls. A surprising finding is that glucose tolerance in DGKE-KO mice is enhanced further at 180 days, and exhibits a much improved picture compared to that of WT controls: Plasma glucose kinetics in DGKE-KO mice at 
180 days of HFD feeding is close to that observed in WT controls under regular chow feeding.

What sorts of changes are visible in WAT during HFD feeding? Epididymal WAT represents visceral WAT. It is useful to monitor changes in mass because it is demarcated clearly from surrounding tissues. In comparison, epididymal WAT mass shows no difference between WT and DGKE-KO mice under regular chow feeding. After 40 days of HFD feeding, however, the WAT mass in DGKE-KO mice increases approximately twofold compared with that in WT mice. In sharp contrast to short-term (40 days) HFD feeding, it is noteworthy that under prolonged HFD feeding such as 90 and 180 days, the WAT mass in DGKE-KO mice decreases by nearly half in WT mice. Histological examination reveals that epididymal WAT is filled mainly with unilocular white adipocytes throughout the course of HFD feeding in WT mice. However, in DGKE-deficient WAT, UCP1-positive cells having multilocular vacuoles in abundant cytoplasm and round nuclei are scattered throughout the tissue at 90 and 180 days. Since UCP1 is a marker for beige/brown adipocyte (Harms and Seale, 2013), those findings suggest that beige adipogenesis or browning of white adipocytes is induced in DGKe-deficient WAT under long-term HFD feeding. Taking glucose tolerance data and histological findings together, a hypothesis can be proposed: In DGKE-KO mice under long-term HFD feeding, beige adipogenesis contributes to efficient energy dissipation, which enhances glucose tolerance. This hypothesis is supported by an earlier study showing that absence of functional beige adipocytes renders mice prone to obesity, insulin resistance, and hepatic steatosis upon HFD feeding (Cohen et al., 2014).

\section{DISCUSSION}

High fat diet studies reveal that under long-term (90 days ） HFD feeding conditions, beige adipogenesis is induced in white adipose tissue, which may contribute to enhanced glucose tolerance in $\mathrm{DGK} \varepsilon-\mathrm{KO}$ mice. Should $\mathrm{DGK} \varepsilon$ be a therapeutic target for obesity? It is not so simple because DGKE-KO mice show severe obesity and insulin resistant phenotype under short-term (40 days) HFD feeding conditions.

\section{REFERENCES}

Bayindir, I., Babaeikelishomi, R., Kocanova, S., Sousa, I. S., Lerch, S., Hardt, O., et al. (2015). Transcriptional Pathways in cPGI2-induced adipocyte progenitor activation for browning. Front. Endocrinol. 6:129. doi: 10.3389/fendo.2015. 00129

Benoit, B., Plaisancie, P., Awada, M., Geloen, A., Estienne, M., Capel, F., et al. (2013). High-fat diet action on adiposity, inflammation, and insulin sensitivity depends on the control low-fat diet. Nutr. Res. 33, 952-960. doi: 10.1016/j. nutres.2013.07.017

Bisogno, T., Howell, F., Williams, G., Minassi, A., Cascio, M. G., Ligresti, A., et al. (2003). Cloning of the first sn1-DAG lipases points to the spatial and temporal regulation of endocannabinoid signaling in the brain. J. Cell Biol. 163, 463-468. doi: $10.1083 /$ jcb.200305129

Blunsom, N. J., and Cockcroft, S. (2020). Phosphatidylinositol synthesis at the endoplasmic reticulum. Biochim. Biophys. Acta Mol. Cell Biol. Lipids 1865:158471. doi: 10.1016/j.bbalip.2019.05.015
In addition, several questions remain unsolved, although regulatory roles of DGK $\varepsilon$ in adipose tissues have been elucidated gradually. First, glucose and fatty acid uptake is apparently facilitated in DGK $\varepsilon-\mathrm{KO}$ mice on regular chow. How does DGK $\varepsilon$ regulate energy uptake? Second, protein expression of ATGL, a TG lipolytic enzyme, is downregulated under obese conditions. In this case, ATGL mRNA level remains unchanged in regular chow and HFD feedings (Nakano et al., 2018). Is ATGL protein degradation promoted in the absence of DGKe? Third, beige adipogenesis is induced in DGKE-deficient WAT under long-term HFD feeding. Is this beiging merely a homeostatic reaction against excess energy accumulation to dissipate extra energy? Or is it regulated directly by DGK $\varepsilon$ ? Further studies must be conducted to address these points.

The primary function of beige/brown adipocytes is thermogenesis. These cells consume glucose and fatty acids to generate heat, instead of ATP production. Results of recent studies suggest that cold exposure facilitates thermogenesis by beige adipogenesis and browning of white adipocytes, in which UCP1 regulates uncoupled respiration to generate heat (Vegiopoulos et al., 2010; Bayindir et al., 2015). Examination of how beiging and browning are regulated by DGK $\varepsilon$ on exposure to cold is expected to be interesting.

\section{AUTHOR CONTRIBUTIONS}

$\mathrm{TN}$ performed the experiments in the original manuscript and summarized the results for the mini review. TN and KG constituted and wrote the manuscript. Both authors contributed to the article and approved the submitted version.

\section{FUNDING}

This work was supported by Grants-in-Aid form the Ministry of Education, Culture, Sports, Science and Technology (MEXT) of Japan [Grant Numbers 20K07239 and 17K08508 (TN)].

Cantley, J. L., Yoshimura, T., Camporez, J. P., Zhang, D., Jornayvaz, F. R., Kumashiro, N., et al. (2013). CGI-58 knockdown sequesters diacylglycerols in lipid droplets/ER-preventing diacylglycerol-mediated hepatic insulin resistance. Proc. Natl. Acad. Sci. USA 110, 1869-1874. doi: $10.1073 /$ pnas. 1219456110

Cinti, S., Mitchell, G., Barbatelli, G., Murano, I., Ceresi, E., Faloia, E., et al. (2005). Adipocyte death defines macrophage localization and function in adipose tissue of obese mice and humans. J. Lipid Res. 46, 2347-2355. doi: 10.1194/jlr. M500294-JLR200

Cohen, P., Levy, J. D., Zhang, Y., Frontini, A., Kolodin, D. P., and Svensson, K. J. (2014). Ablation of PRDM16 and beige adipose causes metabolic dysfunction and a subcutaneous to visceral fat switch. Cell 156, 304-316. doi: 10.1016/j.cell. 2013.12.021

Glomset, J. A. (1996). "A branched metabolic pathway in animal cells converts 2-monoacylglycerol into sn-1-stearoyl-2-arachdonoyl phosphatidylinositol and other phosphoglycerides," in Advances in lipobiology, ed. R. W. Cross (Greemwich, CT: JAI Press), 61-100. 
Goto, K., Hozumi, Y., Nakano, T., Saino, S. S., and Kondo, H. (2007). Cell biology and pathophysiology of the diacylglycerol kinase family: morphological aspects in tissues and organs. Int. Rev. Cytol. 264, 25-63. doi: 10.1016/S0074-7696(07) 64002-9

Goto, K., Tanaka, T., Nakano, T., Okada, M., Hozumi, Y., Topham, M. K., et al. (2014). DGKzeta under stress conditions: "to be nuclear or cytoplasmic, that is the question". Adv. Biol. Regul. 54, 242-253. doi: 10.1016/j.jbior.2013.08.007

Harms, M., and Seale, P. (2013). Brown and beige fat: development, function and therapeutic potential. Nat. Med. 19, 1252-1263. doi: 10.1038/nm.3361

Hozumi, Y., Fujiwara, H., Kaneko, K., Fujii, S., Topham, M. K., Watanabe, M., et al. (2017). Diacylglycerol kinase epsilon localizes to subsurface cisterns of cerebellar Purkinje cells. Cell. Tissue Res. 368, 441-458. doi: 10.1007/s00441017-2579-y

Kanoh, H., Yamada, K., and Sakane, F. (1990). Diacylglycerol kinase: a key modulator of signal transduction? Trends. Biochem. Sci. 15, 47-50. doi: 10.1016/ 0968-0004(90)90172-8

Kim, J. K., Fillmore, J. J., Sunshine, M. J., Albrecht, B., Higashimori, T., Kim, D. W., et al. (2004). PKC-theta knockout mice are protected from fat-induced insulin resistance. J. Clin. Invest. 114, 823-827. doi: 10.1172/JCI22230

Kobayashi, N., Hozumi, Y., Ito, T., Hosoya, T., Kondo, H., and Goto, K. (2007). Differential subcellular targeting and activity-dependent subcellular localization of diacylglycerol kinase isozymes in transfected cells. Eur. J. Cell Biol. 86, 433-444. doi: 10.1016/j.ejcb.2007.05.002

Kolditz, C. I., and Langin, D. (2010). Adipose tissue lipolysis. Curr. Opin. Clin. Nutr. Metab. Care 13, 377-381. doi: 10.1097/MCO.0b013e32833bed6a

Lee, H. Y., Birkenfeld, A. L., Jornayvaz, F. R., Jurczak, M. J., Kanda, S., Popov, V., et al. (2011). Apolipoprotein CIII overexpressing mice are predisposed to diet-induced hepatic steatosis and hepatic insulin resistance. Hepatology 54, 1650-1660. doi: 10.1002/hep.24571

Lemaire, M., Frémeaux-Bacchi, V., Schaefer, F., Choi, M., Tang, W. H., Le Quintrec, M., et al. (2013). Recessive mutations in DGKE cause atypical hemolytic-uremic syndrome. Nat. Genet. 45, 531-536. doi: 10.1038/ng.2590

Li, S. Y., Liu, Y., Sigmon, V. K., McCort, A., and Ren, J. (2005). High-fat diet enhances visceral advanced glycation end products, nuclear O-Glc-Nac modification, p38 mitogen-activated protein kinase activation and apoptosis. Diab. Obes. Metab. 7, 448-454. doi: 10.1111/j.1463-1326.2004.00387.x

Lumeng, C. N., Bodzin, J. L., and Saltiel, A. R. (2007). Obesity induces a phenotypic switch in adipose tissue macrophage polarization. J. Clin. Invest. 117, 175-184. doi: 10.1172/JCI29881

Mannerås-Holm, L., Schönke, M., Brozinick, J. T., Vetterli, L., Bui, H. H., Sanders, P., et al. (2017). Diacylglycerol kinase $\varepsilon$ deficiency preserves glucose tolerance and modulates lipid metabolism in obese mice. J. Lipid. Res. 58, 907-915. doi: 10.1194/jlr.M074443

Matsui, H., Hozumi, Y., Tanaka, T., Okada, M., Nakano, T., Suzuki, Y., et al. (2014). Role of the N-terminal hydrophobic residues of DGKepsilon in targeting the endoplasmic reticulum. Biochim. Biophys. Acta 1842, 1440-1450. doi: 10.1016/ j.bbalip.2014.07.007

Medina, E. A., Afsari, R. R., Ravid, T., Castillo, S. S., Erickson, K. L., and Goldkorn, T. (2005). Tumor necrosis factor-\{alpha\} decreases Akt protein levels in 3T3-L1 adipocytes via the caspase-dependent ubiquitination of Akt. Endocrinology 146, 2726-2735. doi: 10.1210/en.2004-1074

Mérida, I., Arranz-Nicolás, J., Rodríguez-Rodríguez, C., and Ávila-Flores, A. (2019). Diacylglycerol kinase control of protein kinase C. Biochem. J. 476, 1205-1219. doi: 10.1042/BCJ20180620

Nakano, T., Matsui, H., Tanaka, T., Hozumi, Y., Iseki, K., Kawamae, K., et al. (2016). Arachidonoyl-Specific Diacylglycerol Kinase epsilon and the Endoplasmic Reticulum. Front. Cell Dev. Biol. 4:132. doi: 10.3389/fcell.2016.00132

Nakano, T., Seino, K., Wakabayashi, I., Stafforini, D. M., Topham, M. K., and Goto, K. (2018). Deletion of diacylglycerol kinase epsilon confers susceptibility to obesity via reduced lipolytic activity in murine adipocytes. FASEB J. 32, 4121-4131. doi: 10.1096/fj.201701050R

Nakano, T., Topham, M. K., and Goto, K. (2020). Mice lacking DGKe show increased beige adipogenesis in visceral white adipose tissue after long-term high fat diet in a COX-2- dependent manner. Adv. Biol. Regul. 75:100659. doi: 10.1016/j.jbior.2019.100659
Nishizuka, Y. (1992). Intracellular signaling by hydrolysis of phospholipids and activation of protein kinase C. Science 258, 607-614. doi: 10.1126/science. 1411571

Rodriguez de Turco, E. B., Tang, W., Topham, M. K., Sakane, F., Marcheselli, V. L., Chen, C., et al. (2001). Diacylglycerol kinase epsilon regulates seizure susceptibility and long-term potentiation through arachidonoyl- inositol lipid signaling. Proc. Natl. Acad. Sci. USA 98, 4740-4745. doi: 10.1073/pnas. 081536298

Sakane, F., Imai, S., Kai, M., Yasuda, S., and Kanoh, H. (2007). Diacylglycerol kinases: why so many of them? Biochim. Biophys. Acta 1771, 793-806. doi: 10.1016/j.bbalip.2007.04.006

Sakane, F., Mizuno, S., Takahashi, D., and Sakai, H. (2018). Where do substrates of diacylglycerol kinases come from? Diacylglycerol kinases utilize diacylglycerol species supplied from phosphatidylinositol turnover-independent pathways. Adv. Biol. Regul. 67, 101-108. doi: 10.1016/j.jbior.2017.09.003

Samuel, V. T., and Shulman, G. I. (2012). Mechanisms for insulin resistance: common threads and missing links. Cell 148, 852-871. doi: 10.1016/j.cell.2012. 02.017

Tanimura, A., Yamazaki, M., Hashimotodani, Y., Uchigashima, M., Kawata, S., Abe, M., et al. (2010). The endocannabinoid 2-arachidonoylglycerol produced by diacylglycerol lipase alpha mediates retrograde suppression of synaptic transmission. Neuron 65, 320-327. doi: 10.1016/j.neuron.2010.01.021

Tang, W., Bunting, M., Zimmerman, G. A., McIntyre, T. M., and Prescott, S. M. (1996). Molecular cloning of a novel human diacylglycerol kinase highly selective for arachidonate-containing substrates. J. Biol. Chem. 271, 1023710241. doi: 10.1074/jbc.271.17.10237

Topham, M. K., and Epand, R. M. (2009). Mammalian diacylglycerol kinases: molecular interactions and biological functions of selected isoforms. Biochim. Biophys. Acta 1790, 416-424. doi: 10.1016/j.bbagen.2009.01.010

Vegiopoulos, A., Muller-Decker, K., Strzoda, D., Schmitt, I., Chichelnitskiy, E., Ostertag, A., et al. (2010). Cyclooxygenase-2 controls energy homeostasis in mice by de novo recruitment of brown adipocytes. Science 328, 1158-1161. doi: 10.1126/science.1186034

Wang, S., Soni, K. G., Semache, M., Casavant, S., Fortier, M., Pan, L., et al. (2008). Lipolysis and the integrated physiology of lipid energy metabolism. Mol. Genet. Metab. 95, 117-126. doi: 10.1016/j.ymgme.2008.06.012

Yamamoto, M., Tanaka, T., Hozumi, Y., Saino-Saito, S., Nakano, T., Tajima, K., et al. (2014). Expression of mRNAs for the diacylglycerol kinase family in immune cells during an inflammatory reaction. Biomed. Res. 35, 61-68. doi: 10.2220/biomedres.35.61

Zechner, R., Zimmermann, R., Eichmann, T. O., Kohlwein, S. D., Haemmerle, G., et al. (2012). FAT SIGNALS-lipases and lipolysis in lipid metabolism and signaling. Cell Metab. 15, 279-291. doi: 10.1016/j.cmet.2011.1 2.018

Zhu, J., Chaki, M., Lu, D., Ren, C., Wang, S. S., Rauhauser, A., et al. (2016). Loss of diacylglycerol kinase epsilon in mice causes endothelial distress and impairs glomerular Cox-2 and PGE2 production. Am. J. Physiol. Renal Physiol. 310, F895-F908. doi: 10.1152/ajprenal.00431.2015

Conflict of Interest: The authors declare that the research was conducted in the absence of any commercial or financial relationships that could be construed as a potential conflict of interest.

Publisher's Note: All claims expressed in this article are solely those of the authors and do not necessarily represent those of their affiliated organizations, or those of the publisher, the editors and the reviewers. Any product that may be evaluated in this article, or claim that may be made by its manufacturer, is not guaranteed or endorsed by the publisher.

Copyright (c) 2022 Nakano and Goto. This is an open-access article distributed under the terms of the Creative Commons Attribution License (CC BY). The use, distribution or reproduction in other forums is permitted, provided the original author(s) and the copyright owner(s) are credited and that the original publication in this journal is cited, in accordance with accepted academic practice. No use, distribution or reproduction is permitted which does not comply with these terms. 